\title{
Therapeutic Outcome of $>10$ Cycles of Cabazitaxel for Castration-resistant Prostate Cancer: A Multi-institutional Study
}

\author{
MASAKI SHIOTA ${ }^{1}$, MOTONOBU NAKAMURA ${ }^{2}$, AKIRA YOKOMIZO ${ }^{3}$, TOSHIHISA TOMODA ${ }^{4}$, \\ NAOTAKA SAKAMOTO ${ }^{5}$, NARIHITO SEKI ${ }^{6}$, SHUJI HASEGAWA ${ }^{7}$, TAKAKAZU YUNOKI ${ }^{8}$, \\ MASAHIKO HARANO ${ }^{9}$, KENTARO KUROIWA $^{10}$ and MASATOSHI ETO ${ }^{1}$ \\ ${ }^{1}$ Department of Urology, Graduate School of Medical Sciences, Kyushu University, Fukuoka, Japan; \\ ${ }^{2}$ Department of Urology, National Hospital Organization Kyushu Cancer Center, Fukuoka, Japan; \\ ${ }^{3}$ Division of Urology, Harasanshin Hospital, Fukuoka, Japan; \\ ${ }^{4}$ Department of Urology, Oita Prefectural Hospital, Oita, Japan; \\ ${ }^{5}$ Department of Urology, National Hospital Organization Kyushu Medical Center, Fukuoka, Japan; \\ ${ }^{6}$ Department of Urology, Kyushu Central Hospital, Fukuoka, Japan; \\ ${ }^{7}$ Department of Urology, Kitakyushu Municipal Medical Center, Kitakyushu, Japan; \\ ${ }^{8}$ Department of Urology, Japanese Red Cross Fukuoka Hospital, Fukuoka, Japan; \\ ${ }^{9}$ Department of Urology, JCHO Kyushu Hospital, Kitakyushu, Japan; \\ ${ }^{10}$ Department of Urology, Miyazaki Prefectural Miyazaki Hospital, Miyazaki, Japan
}

\begin{abstract}
Background/Aim: Cabazitaxel use has usually been limited to up to 10 cycles in most countries according to the protocol in the TROPIC trial. Therefore, clinical data on cabazitaxel use beyond 10 cycles is limited. The aim of this study was to report the therapeutic outcome of cabazitaxel chemotherapy administered for $>10$ cycles. Patients and Methods: This study included 74 Japanese patients with prostate cancer between 2014 and 2017. Patients background, and treatment outcomes including PSA decline, progressionfree survival, treatment-failure-free survival, overall survival, and adverse events were investigated, comparing patients treated with $\leq 10$ and $>10$ cycles. Results: Patients characteristics were favorable as indicated by the higher number of cycles of prior docetaxel chemotherapy, absence of pain, and absence of bony and visceral metastases among men who received $>10$ cycles of cabazitaxel. PSA response, progression-free survival,
\end{abstract}

Correspondence to: Masaki Shiota MD, Ph.D., Department of Urology, Graduate School of Medical Sciences, Kyushu University, 3-1-1 Maidashi, Higashi-ku, Fukuoka 812-8582, Japan. Tel: +81 926425603, Fax: +81 926425618, e-mail: shiota@uro.med.kyushuu.ac.jp

Key Words: Castration-resistant prostate cancer, cabazitaxel, outcome, adverse event. treatment-failure-free survival and overall survival were better among patients treated with $>10$ cycles of cabazitaxel compared to those treated with $\leq 10$ cycles. The incidence of severe adverse events was similar between the two groups. Conclusion: Taken together, this study suggested that continuous chemotherapy with cabazitaxel beyond 10 cycles may be beneficial.

The phase III TROPIC trial has shown that cabazitaxel, the next-generation taxane, after docetaxel chemotherapy, prolongs survival of patients suffering from castration-resistant prostate cancer (CRPC) and provides pain relief $(1,2)$. Usually, clinical use of cabazitaxel has been limited to up to 10 cycles in most countries according to the protocol in the TROPIC trial (1). However, it can be hypothesized that cabazitaxel use beyond 10 cycles may be more beneficial to patients with CRPC if antitumor effects are sustained and toxicities are tolerable. In Japan, cabazitaxel is being used for more than 10 cycles. Therefore, in this study, we aimed to reveal the therapeutic outcome of long-term cabazitaxel chemotherapy, comparing patients treated with $\leq 10$ and $>10$ cycles.

\section{Patients and Methods}

Patients. This study retrospectively enrolled Japanese men treated with cabazitaxel between 2014 and 2017 at the following institutions: Kyushu University Hospital (Fukuoka), National Hospital Organization Kyushu Cancer Center (Fukuoka), Harasanshin Hospital (Fukuoka), Oita Prefectural Hospital (Oita), 
Table I. Patients' characteristics according to number of cycles of cabazitaxel.

\begin{tabular}{|c|c|c|c|c|}
\hline \multirow[t]{2}{*}{ Variables } & \multicolumn{3}{|c|}{ Cycle number of cabazitaxel } & \multirow[b]{2}{*}{$p$-Value } \\
\hline & All $(n=74)$ & $\leq 10(\mathrm{n}=62)$ & $>10(\mathrm{n}=12)$ & \\
\hline Median age, years (IQR) & $72(67-76)$ & $72(67-76)$ & $74(64-77)$ & 0.81 \\
\hline Median PSA at diagnosis, $\mathrm{ng} / \mathrm{ml}$ (IQR) & $48.3(19.4-376.8)$ & $46.2(18.4-349.7)$ & $192(34.0-645.7)$ & 0.21 \\
\hline NA & 2 & 2 & 0 & \\
\hline \multicolumn{5}{|l|}{ Biopsy Gleason score, $\mathrm{n}(\%)$} \\
\hline$\leq 7$ & $12(16.9 \%)$ & $9(15.3 \%)$ & $3(25.0 \%)$ & \\
\hline 8 & $12(16.9 \%)$ & $10(16.9 \%)$ & $2(16.7 \%)$ & \\
\hline$\geq 9$ & $47(66.2 \%)$ & $40(67.8 \%)$ & $7(58.3 \%)$ & 0.71 \\
\hline NA & 3 & 3 & 0 & \\
\hline \multicolumn{5}{|l|}{ Prior local therapy, n (\%) } \\
\hline Absence & $53(71.6 \%)$ & $43(69.4 \%)$ & $10(83.3 \%)$ & \\
\hline Presence & $21(28.4 \%)$ & $19(30.6 \%)$ & $2(16.7 \%)$ & 0.33 \\
\hline Time to CRPC, years (IQR) & $1.3(0.7-2.4)$ & $1.3(0.7-2.3)$ & $1.7(0.7-4.2)$ & 0.31 \\
\hline NA & 7 & 7 & 0 & \\
\hline Cycle number of prior docetaxel & $8(5-12)$ & $7(5-10)$ & $13(7-20)$ & $0.0092 *$ \\
\hline \multicolumn{5}{|l|}{ Prior treatment for CRPC, $\mathrm{n}(\%)$} \\
\hline Abiraterone/enzalutamide & $62(83.8 \%)$ & $52(83.4 \%)$ & $10(83.3 \%)$ & 0.96 \\
\hline Radium-223 & $4(5.4 \%)$ & $4(6.5 \%)$ & $0(0.0 \%)$ & 0.37 \\
\hline \multicolumn{5}{|l|}{ ECOG PS at pre-treatment, $\mathrm{n}(\%)$} \\
\hline 0 & $43(65.2 \%)$ & $33(61.1 \%)$ & $10(83.3 \%)$ & \\
\hline 1 & $15(22.7 \%)$ & $13(24.1 \%)$ & $2(16.7 \%)$ & \\
\hline$\geq 2$ & $8(12.1 \%)$ & $8(14.8 \%)$ & $0(0.0 \%)$ & 0.12 \\
\hline NA & 8 & 8 & 0 & \\
\hline \multicolumn{5}{|l|}{ Pain at pre-treatment, $\mathrm{n}(\%)$} \\
\hline Absence & $37(50.0 \%)$ & $27(43.5 \%)$ & $10(83.3 \%)$ & \\
\hline Presence & $37(50.0 \%)$ & $35(56.5 \%)$ & $2(16.7 \%)$ & $0.012 *$ \\
\hline Median PSA at pre-treatment, $\mathrm{ng} / \mathrm{ml}$ (IQR) & $72.3(18.0-240.6)$ & $93.1(17.3-293.2)$ & $38.8(28.8-77.6)$ & 0.22 \\
\hline \multicolumn{5}{|l|}{ Metastatic sites, n (\%) } \\
\hline Lymph node & $43(58.1 \%)$ & $37(59.7 \%)$ & $6(50.0 \%)$ & 0.53 \\
\hline Bone & $66(89.2 \%)$ & $58(93.5 \%)$ & $8(66.7 \%)$ & $0.0061 *$ \\
\hline Visceral & $20(27.0 \%)$ & $20(32.3 \%)$ & $0(0.0 \%)$ & $0.021 *$ \\
\hline
\end{tabular}

*Statistically significant. IQR: Interquartile range; NA: not available; PS: performance status.

Table II. Grade $\geq 3$ adverse events according to number of cycles of cabazitaxel.

\begin{tabular}{|c|c|c|c|c|}
\hline & \multicolumn{3}{|c|}{ Cycle number of cabazitaxel } & \multirow[b]{2}{*}{$p$-Value } \\
\hline & All $(n=74)$ & $\leq 10(n=62)$ & $>10(\mathrm{n}=12)$ & \\
\hline \multicolumn{5}{|l|}{ Hematological } \\
\hline Neutropenia $(\geq \mathrm{G} 3$ ) & $54(73.0 \%)$ & $45(72.6 \%)$ & $9(75.0 \%)$ & 0.86 \\
\hline Febrile neutropenia $(\geq \mathrm{G} 3$ ) & $23(31.1 \%)$ & $20(32.3 \%)$ & $3(25.0 \%)$ & 0.62 \\
\hline Non-hematological $(\geq \mathrm{G} 3)$ & $17(23.0 \%)$ & $14(22.6 \%)$ & $3(25.0 \%)$ & 0.86 \\
\hline Non-hematological (G5) & $5(6.8 \%)$ & $5(8.1 \%)$ & $0(0.0 \%)$ & 0.31 \\
\hline
\end{tabular}

National Hospital Organization Kyushu Medical Center (Fukuoka), Kyushu Central Hospital (Fukuoka), Kitakyushu Municipal Medical Center (Kitakyushu), Japanese Red Cross Fukuoka Hospital (Fukuoka), JCHO Kyushu Hospital (Kitakyushu), and Miyazaki Prefectural Miyazaki Hospital (Miyazaki). The Institutional Review Boards approved this study. Inclusion criteria are as follows: (i) histopathological diagnosis as carcinoma of the prostate; (ii) age $\geq 20$ years; and (iii) progression despite primary androgendeprivation therapy.

Treatment. Cabazitaxel $\left(20-25 \mathrm{mg} / \mathrm{m}^{2}\right)$ was administered as a 3- or 4-weekly regimen according to the physician's preference $(1,3)$, 
A

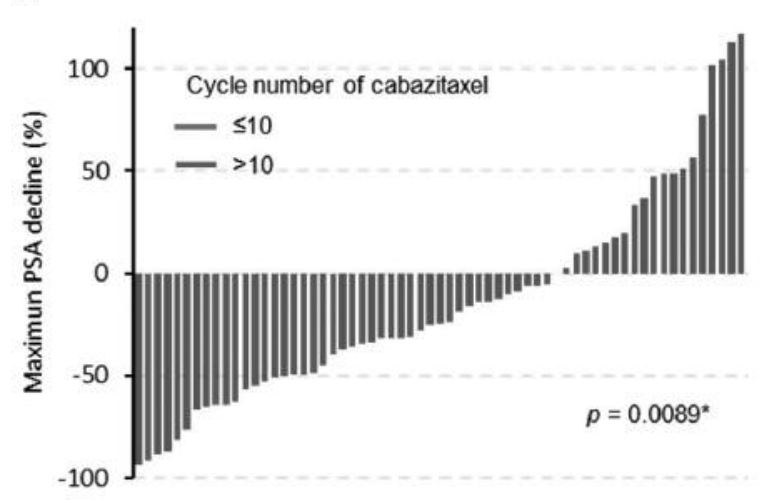

B

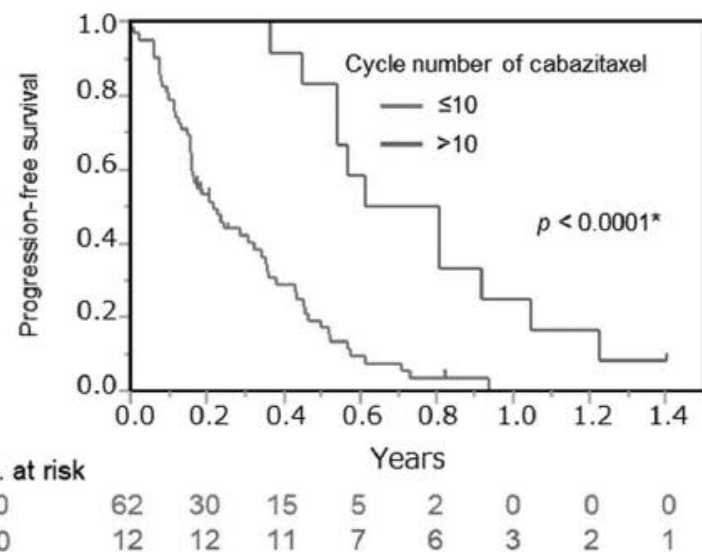

D

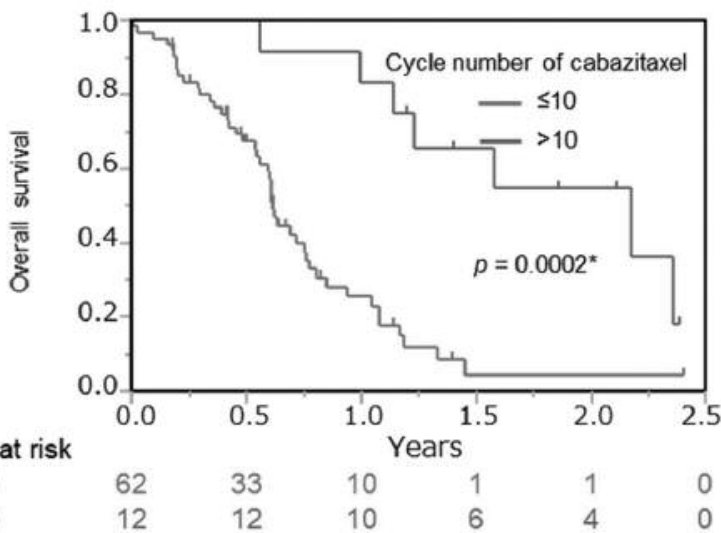

Figure 1. Anticancer effects of cabazitaxel chemotherapy administered in $\leq 10$ or $>10$ cycles. Waterfall plots showing greatest decline in PSA levels from baseline $(A)$, progression-free survival $(B)$, treatment-failure-free survival $(C)$, and overall survival $(D)$ in 74 patients with $C R P C$ who received $\leq 10$ or $>10$ cycles of cabazitaxel chemotherapy.

and only one case was treated with $15 \mathrm{mg} / \mathrm{m}^{2}$ cabazitaxel. Prednisolone $5 \mathrm{mg}$ was given twice daily simultaneously with medical or surgical castration.

Measurements. Disease progression was defined according to PCWG2 (4). Treatment failure was defined as discontinuation of cabazitaxel, which was determined by disease progression and adverse events (AEs), or patient refusal. AEs were assessed according to the National Cancer Institute Common Terminology Criteria for Adverse Events version 4.0. Pain and performance status were determined by the prescription of analgesics for the symptom and according to the Eastern Cooperative Oncology Group criteria, respectively.

Statistical analysis. All statistical analyses were performed using JMP13 (SAS Institute). The differences of categorical and continuous variables were examined by Pearson's chi square test and Wilcoxon rank sum, respectively. The Kaplan-Meier method and log-rank test were used for survival analyses. $p<0.05$ was considered significant.

\section{Results}

A median of four (interquartile range=2-8) cycles of cabazitaxel therapy were administered to 74 patients according to the physician's judgement and patient's preference. Patients' backgrounds are shown in Table I. More cycles of prior docetaxel chemotherapy, absence of pain, and absence of bony and visceral metastases were recognized among men treated with $>10$ cycles of cabazitaxel (Table I), which suggested more favorable characteristics. Not surprisingly, PSA response, progression-free survival, treatment-failure-free survival and overall survival were all better among patients treated with $>10$ cycles compared to those treated with $\leq 10$ cycles of cabazitaxel (Figure 1 ). The incidence of Grade $\geq 3$ AEs was similar between patients treated with $\leq 10$ and $>10$ cycles of cabazitaxel (Table II). Notably, lethal AEs did not occur in patients with $>10$ cycles of cabazitaxel, while pulmonary emboli, peripheral neuritis, 
and macular edema were observed in each case, leading to discontinuation of cabazitaxel chemotherapy.

\section{Discussion}

In the present study, more cycles of prior docetaxel, absence of pain, and absence of bony and visceral metastases were associated with $>10$ cycles of cabazitaxel chemotherapy, suggesting that they are favorable prognostic factors for cabazitaxel chemotherapy and lead to successful long-term cabazitaxel treatment. Consistently, Halabi et al. have previously reported several prognostic factors for overall survival using data from the TROPIC trial, and pain and short response to docetaxel were identified as adverse prognostic factors (5).

Our study showed cabazitaxel also exerted excellent anticancer activity and modestly increased cumulative toxicity even when $>10$ cycles were administered. Intriguingly, therapeutic outcomes of cabazitaxel rechallenge have been reported, showing moderate anticancer effects with PSA response in $24 \%$ of cases and 13.7 months survival without cumulative toxicity $(6,7)$. This agrees with the excellent outcomes among patients who received $>10$ cycles of cabazitaxel in the present study.

\section{Conclusion}

These results support continuous chemotherapy with cabazitaxel for $>10$ cycles if disease control is achieved, there is less cumulative toxicity and patients wish to continue cabazitaxel chemotherapy.

\section{Conflicts of Interest}

The Authors declare no conflicts of interest regarding this study.

\section{Authors' Contributions}

MS designed the study, analyzed the data, and wrote the draft of the manuscript. All other authors have contributed to data collection and interpretation, and critically reviewed the manuscript. EM supervised the study.

\section{Acknowledgements}

This work was supported by JSPS KAKENHI grant (17K11145). The Authors would like to acknowledge the contributions of the following collaborators: Akito Yamaguchi at Harasanshin Hospital (Fukuoka), Takashi Dejima at Kyushu Central Hospital (Fukuoka), Satoshi Otsubo at Kitakyushu Municipal Medical Center (Kitakyushu), Akio Tsutsui at JCHO Kyushu Hospital (Kitakyushu), and Yoshifumi Hori at Miyazaki Prefectural Miyazaki Hospital (Miyazaki). The Authors also thank Edanz Group (www.edanzediting.com/ac) for editing a draft of this manuscript.

\section{References}

1 de Bono JS, Oudard S, Ozguroglu M, Hansen S, Machiels JP, Kocak I, Gravis G, Bodrogi I, Mackenzie MJ, Shen L, Roessner M, Gupta S and Sartor AO; TROPIC Investigators: Prednisone plus cabazitaxel or mitoxantrone for metastatic castrationresistant prostate cancer progressing after docetaxel treatment: a randomised open-label trial. Lancet 376: 1147-1154, 2010. PMID: 20888992. DOI: 10.1016/S0140-6736(10)61389-X

2 Bahl A, Oudard S, Tombal B, Ozgüroglu M, Hansen S, Kocak I, Gravis G, Devin J, Shen L, de Bono JS and Sartor AO; TROPIC Investigators: Impact of cabazitaxel on 2-year survival and palliation of tumour-related pain in men with metastatic castration-resistant prostate cancer treated in the TROPIC trial. Ann Oncol 24: 2402-2408, 2013. PMID: 23723295. DOI: 10.1093/annonc/mdt194

3 Eisenberger M, Hardy-Bessard AC, Kim CS, Géczi L, Ford D, Mourey L, Carles J, Parente P, Font A, Kacso G, Chadjaa M, Zhang W, Bernard J and de Bono J: Phase III study comparing a reduced dose of cabazitaxel $\left(20 \mathrm{mg} / \mathrm{m}^{2}\right)$ and the currently approved dose $\left(25 \mathrm{mg} / \mathrm{m}^{2}\right)$ in postdocetaxel patients with metastatic castration-resistant prostate cancer-PROSELICA. J Clin Oncol 35: 3198-3206, 2017. PMID: 28809610. DOI: 10.1200/JCO.2016.72.1076

4 Scher HI, Halabi S, Tannock I, Morris M, Sternberg CN, Carducci MA, Eisenberger MA, Higano C, Bubley GJ, Dreicer R, Petrylak D, Kantoff P, Basch E, Kelly WK, Figg WD, Small EJ, Beer TM, Wilding G, Martin A and Hussain M; Prostate Cancer Clinical Trials Working Group: Design and end points of clinical trials for patients with progressive prostate cancer and castrate levels of testosterone: recommendations of the Prostate Cancer Clinical Trials Working Group. J Clin Oncol 26: 11481159, 2008. PMID: 18309951. DOI: 10.1200/JCO.2007.12.4487

5 Halabi S, Lin CY, Small EJ, Armstrong AJ, Kaplan EB, Petrylak D, Sternberg CN, Shen L, Oudard S, de Bono J and Sartor O: Prognostic model predicting metastatic castration-resistant prostate cancer survival in men treated with second-line chemotherapy. J Natl Cancer Inst 105: 1729-1737, 2013. PMID: 24136890. DOI: $10.1093 /$ jnci/djt280

6 Thibault C, Eymard JC, Birtle A, Krainer M, Baciarello G, Fléchon A, Le Moulec S, Spaeth D, Laguerre B, Caffo O, Deville JL, Beuzeboc P, Hasbini A, Gross-Goupil M, Helissey C, Bennamoun M, Hardy-Bessard AC and Oudard S: Efficacy of cabazitaxel rechallenge in heavily treated patients with metastatic castration-resistant prostate cancer. Eur J Cancer 97: 41-48, 2018. PMID: 29636272. DOI: 10.1016/j.ejca.2018.03.008

7 Di Lorenzo G, Bracarda S, Gasparro D, Gernone A, Messina C, Zagonel V, Puglia L, Bosso D, Dondi D, Sonpavde G, Lucarelli G, De Placido S and Buonerba C: Lack of cumulative toxicity associated with cabazitaxel use in prostate cancer. Medicine 95: e2299, 2016. PMID: 26765406. DOI: 10.1097/MD.000000000 0002299 